CLASSIC PAPER

\title{
latrogenic illness on a general medical service at a university hospital ${ }^{*}$
}

\author{
K Steel, P M Gertman, C Crescenzi, J Anderson
}

Qual Saf Health Care 2004;13:76-81. doi: 10.1136/qshc.2002.003830

We found that $36 \%$ of 815 consecutive patients on a general medical service of a university hospital had an iatrogenic illness. In $9 \%$ of all persons admitted, the incident was considered major in that it threatened life or produced considerable disability. In $2 \%$ of the 815 patients, the iatrogenic illness was believed to contribute to the death of the patient. Exposure to drugs was a particularly important factor in determining which patients had complications. Given the increasing number and complexity of diagnostic procedures and therapeutic agents, monitoring of untoward events is essential, and attention should be paid to educational efforts to reduce the risks of iatrogenic illness.

atrogenic illness has been a well recognized phenomenon for over a quarter of a century. In 1955, Barr made note of the price that we pay for modern medical care. ${ }^{1}$ There is a pressing need for a better understanding of the "diseases that would not have occurred if sound therapeutic procedure had not been employed,"'2 as Moser defined the illness of medical progress in addition to illnesses resulting from diagnostic endeavors. ${ }^{3-5}$

The problem is not limited to acute care hospitals, but it may be most dramatically demonstrated there. Schimmel noted in 1964 that $20 \%$ of patients admitted to the medical wards of a teaching hospital over an 8 month period had one or more untoward episodes. ${ }^{4}$ Over the past 15 years the number and complexity of diagnostic efforts has increased markedly, the number of drugs in use has risen yearly, and the patient population has aged.

We undertook a study to re-evaluate the risks of care in one setting: a medical service in a tertiary care hospital. It is emphatically stated that we are making no judgment of the relative risk and benefit of the encounter between the patient and the hospital staff. Furthermore, we are making no comparison between this setting and any other (for example, home care); instead, we are attempting to define the types and magnitudes of risks in a specific setting.

\section{METHODS}

See end of article for authors' affiliations

\section{Study population}

The basic approach was to monitor all new patients admitted to two floors of a medical service at a university teaching hospital for a 5 month period in 1979. These two floors contain
83 beds and include a medical intensive care unit (ICU), a coronary care unit (CCU), a metabolic unit, and two general medical wards. Another medical floor that is predominantly reserved for patients with cancer was excluded from the study because of the recognized high risk of iatrogenic complications in these patients. Periodically, during the course of hospitalization or shortly after discharge, each patient's record was reviewed by a member of the project staff and abstracted according to a standardized 27item instrument. In addition to abstraction of the record for all patients, identification of iatrogenic issues was supplemented with staff questioning of clinical personnel who cared for the patient and with information obtained from utilization review coordinators who were evaluating the patients' needs for continued hospitalization.

\section{Study instrument}

The standardized process of abstraction and review focused on five areas: age and sex, admission data, hospital interventions, discharge data, and complications. Admission factors included the admission source (from the home, senior citizen housing, nursing home, or acute care hospital); admission type (elective or nonelective); admission location (ICU, CCU, or ward); house officer's assessment of clinical condition (critical to poor, fair, or stable to good); level of consciousness (comatose, awake but not oriented, or alert); and medical diagnoses at admission. Hospital interventions were classified according to types of major diagnostic and therapeutic procedures (e.g. cardiac catheterization), number and type of drugs, administration of biologic substances, other therapeutic and management methods, and accession to an experimental research study. Discharge data included the length of stay, diagnoses at discharge, and discharge location (to the home, senior citizen housing, a nursing home, or an acute care hospital; a notation was also made if the patient died or if the discharge location was unknown). Complications were organized according to type (classified into 49 predefined categories), severity (major or minor), immediate cause (classified into 68 predefined intervention categories), and degree of resulting disability; it was also noted whether a complication was judged to have resulted in extension of a patient's hospital stay.

*This is a reprint of a paper that appeared in New England Journal of Medicine, 1981, Volume 304, pages 638-42. 


\section{Criteria for iatrogenic events}

We defined an iatrogenic illness as any illness that resulted from a diagnostic procedure or from any form of therapy. In addition, we included harmful occurrences (for example, injuries from a fall or decubitus ulcers) that were not natural consequences of the patient's diseases. However, the term "iatrogenic" should not be construed to mean that there was any culpability on the part of the physician or hospital, or that the illness was necessarily preventable.

In all but the most extreme examples, determination of an iatrogenic illness is a judgmental process. Because of the implications of these judgments we used a conservative approach in determining their occurrence, frequency, and severity. If there was even slight reason to believe that an event reflected the natural progression of a disease, it was not included. Occurrences that were obviously not serious (such as the simple discomfort and inconvenience of intravenous therapy, delays in scheduling, room changes, and the like) were not recorded. Minor problems that resolved without specific therapy, such as minor changes in electrocardiograms, were dismissed. A complication was recorded as a single event even if a plethora of problems resulted from one intervention. For example, if a drug produced hypotension, renal failure, and congestive heart failure, only one iatrogenic illness was recorded. If no documentation of any sort was available, no iatrogenic illness was recorded despite suspicions of the project staff that one had occurred. This problem was particularly common in cases of apparent psychiatric disturbances. We did not count iatrogenic illnesses that occurred before the patient's admission to one of the two floors or those that occurred after the patient's transfer off the service but before discharge from the hospital. Finally, most persons admitted for treatment of cancer, who were at exceptional risk of iatrogenic complications while they were undergoing chemotherapy and radiation therapy, were excluded from the study, as noted earlier.

Thus, we believe that these criteria are conservative; if the study had judgmental bias, the effect was to underestimate the number of iatrogenic illnesses. The severity of a complication was determined to be major if in the principal investigator's opinion the event was life threatening (e.g. cardiac arrest or renal failure), or if it produced serious disability that was present on the patient's discharge from the hospital.

No attempt was made to grade the seriousness of other iatrogenic illnesses, all of which were recorded as minor. Thus, for example, skin rashes and episodes of vomiting that were probably due to a drug were listed as minor illnesses. Similarly, falls that did not result in fractures but caused only minor lacerations or musculoskeletal pain were included in this category. Nonetheless, it must be noted that in almost all cases the problems were judged by the staff caring for the patient to be of enough importance to warrant documentation in the record.

\section{Statistical procedures}

In investigating simple associations between two variables, we used standard statistical techniques. One way analysis of variance and two sample tests were used in tests for associations between a scalar variable (age, length of stay, or drug exposure) and the occurrence of any major complications. A chi-square test of association was used for the categorical variables relating to discharge location, admission source, clinical status at admission, and admission location.

Further analysis was performed with a logit model. The data for such analyses consisted of sets of counts of patients with and without complications in cells defined by particular combinations of possible values of the categorical factors. If $p$ is the proportion of patients with complications in a particular cell, a linear model for $p$ explains variation in $p$ from cell to cell as a linear function of levels and combinations of levels of various factors. We used a logit model approach in which not $p$ but its $\operatorname{logit}(\log [p / 1-p])$ is the dependent variable in the linear model. This formulation has advantages over a model that is linear in $p$ itself in interpretability of interaction and in the range of variation allowed. ${ }^{6}$

\section{RESULTS}

\section{Frequency and type of iatrogenic events}

A total of 815 patients were seen on the two medical floors during the period of study. Of these, 290 patients (36\%) had one or more iatrogenic illnesses, with a total of 497 such occurrences. One hundred and sixty five patients had one reported complication $(57 \%$ of all patients with complications), and 125 patients (43\%) had between two and seven.

A total of 76 patients ( $9 \%$ of all those admitted) had major complications. (Two additional patients had major complications but, because of incomplete medical records, the data on these patients were not included in the calculations for iatrogenic illness.) In 15 patients ( $2 \%$ of all patients and more than $5 \%$ of those with complications) the iatrogenic illness was believed to have contributed to the death of the patient. Thirty of the 290 patients with iatrogenic illnesses died, as compared with only 33 of the 525 patients with no complications; this difference was significant $(\mathrm{p}<0.05)$.

Table 1 lists the types of hospital interventions that were judged to have caused complications, the number of complications, and the percentage of each grouping that were major complications. The three largest intervention categories (in which a single intervention appeared to be related to a complication) were drugs (208 complications), cardiac catheterizations (45), and falls (35). Of all patients with complications, $53 \%$ had at least one problem related to drug exposure. Furthermore, the count of drug complications is probably an underrepresentation of their true effect. For example, if a patient became dizzy or disoriented at night possibly because of a drug and fell, but we were unable to

Table 1 Hospital interventions leading to iatrogenic complications

\begin{tabular}{lll} 
& \multicolumn{2}{l}{ Complications } \\
\cline { 2 - 3 } Type of intervention & No. & $\%$ Major \\
\hline Drugs & 208 & 19 \\
Nitrates & 26 & 15 \\
Digoxin & 15 & 33 \\
Lidocaine & 15 & 13 \\
Aminophylline & 15 & 7 \\
Quinidine/disopyramide/procainamide & 13 & 15 \\
Heparin/warfarin & 13 & 54 \\
Penicillins & 10 & 10 \\
Benzodiazepines & 10 & 0 \\
Antihypertensives & 10 & 30 \\
Propranolol & 9 & 44 \\
Other and multiple & 72 & 15 \\
Diagnostic and therapeutic procedures & 175 & 28 \\
Cardiac catheterization & 45 & - \\
Intravenous therapy & 34 & - \\
Urinary tract catheterization & 10 & - \\
Other (e.g. arteriography, peritoneal dialysis, & & \\
use of nasogastric tube, Swan-Ganz & 86 & - \\
catheterization, or hemodialysis) & 114 & 21 \\
Miscellaneous & 35 & - \\
Falls & 79 & - \\
Other (e.g. transfusion, diet management, & 79 & 22 \\
transportation, or nursing procedures) & 497 & \\
Total complications & & \\
\hline & & \\
\hline
\end{tabular}


make such an attribution, the event was classified as a fall. If we believed that several different factors contributed to an iatrogenic illness but a judgment could not be reached about which factor was predominant, the event was recorded in the "miscellaneous/other" intervention category.

Table 1 also illustrates that the set of drugs causing iatrogenic disease varied widely, with no single agent appearing to dominate the list. However, patients were frequently taking several pharmacologic agents when an iatrogenic disease occurred. If it was not possible to determine which particular drug was the offending agent, or if it appeared that the event was due to a combination of pharmacologic agents, the "other and multiple" drug category was used. Because of this procedure and because the numbers are small, no comparison with respect to the seriousness of complications should be made between drugs. Nonetheless, serious consequences did seem to occur from the use of almost all groups of drugs listed. Serious arrhythmias, hypotension requiring intervention, and changes in mental state were among such major complications.

The type of complication arising during the hospitalization and the percentage in each category that were major are shown in table 2. Clearly, the list is varied. It should also be noted how few metabolic complications occurred, although only serious ones were noted. Aspiration pneumonia, nosocomial infection, and problems that were secondary to invasive procedures accounted for the majority of the major complications.

\section{Factors associated with iatrogenic events Simple association}

Data in all 815 patients about age, sex, admission source, admission route, admission location, house officer's assessment, level of consciousness, discharge location, drug exposure, and length of stay were analyzed for simple associations with iatrogenic events.

Patients admitted from nursing homes or acute care hospitals were more likely to have complications than were patients admitted from their homes. A house officer's assessment of "critical to poor" condition on admission was also associated with a higher complication rate than was an assessment of "stable to good," and patients admitted to the ICU or CCU had higher complication rates than did patients admitted to the general wards.

Age, drug exposure, and length of stay were each positively associated with a complication $(\mathrm{p}<0.001)$; in addition, total number of drugs and length of stay were each greater in patients with major complications than in patients with minor complications (table 3 ).

Simultaneous effects of admission status variables Through logit analysis, we investigated the extent to which a patient's age, sex, and condition at admission might jointly affect the probability of an iatrogenic complication occurring

Table 2 latrogenic complications in 290 patients

\begin{tabular}{lll}
\hline Type of complication & No. of episodes & \% Major \\
\hline Cardiopulmonary & 102 & 37 \\
Infection or inflammation & 89 & 33 \\
Gastrointestinal & 47 & 9 \\
Neurologic & 43 & 23 \\
Allergic reaction & 35 & 3 \\
Bleeding & 23 & 39 \\
Metabolic & 6 & 100 \\
Miscellaneous & 152 & 11 \\
Total & 497 & 22 \\
\hline
\end{tabular}

in the hospital. The patient's age (under or over 65 years), sex, admission source (from home or from another hospital), and admission location (into the ICU or CCU or into the ward) were considered in this analysis, together with the house officer's assessment of the patient's condition on admission (critical, fair, or stable). Of these variables, only the source of admission and the house officer's assessment of the patient's condition affected the chance of a complication arising during the hospitalization. That is, the patient's age, sex, and admission location did not affect the chance of a complication. Table 4 gives smoothed probabilities obtained from the logit model, with the probability of any complication and the probability of a major complication considered separately. Admission source was associated with the presence of any complication $(\mathrm{p}<0.0001)$ and with major complications $(\mathrm{p}<0.003)$; the house officer's assessment was also significantly related $(\mathrm{p}<0.0001$ for any complication and $\mathrm{p}<0.0006$ for major complications).

Although there was a simple association of iatrogenic complications with the age of the patient, the absence of this factor from the logit model indicates that the effect of chronologic age was secondary. Older patients were more likely to be admitted to the hospital in fair or critical condition than were younger patients (chi-square, 27.2; $\mathrm{p}<0.0001$ with age in the three categories of under 65 years, 65 to 74 , and 75 and over).

\section{Drug use and length of stay}

Since the data on total drug exposure and length of stay were totals for a patient's entire hospital stay, their association with the occurrence of a complication does not in itself indicate a causal role, since an extended hospital stay and exposure to new drugs may also have resulted from the complication.

For the 76 patients with major complications, the average total length of stay was 19.3 days, with averages of 7.8 days before the first major complication and 11.5 days afterward. The average number of new drugs prescribed before the first major complication was 10.7 and, on average, 6.4 new drugs were prescribed after such an event. The number of new drugs prescribed for these patients before the occurrence of major complications was greater than the number of drugs prescribed throughout the entire hospital stay in patients who had no complications $(t=5.66, \mathrm{p}<0.001)$.

\section{DISCUSSION}

These results appear to warrant several observations. First of all, the risk incurred during hospitalization is not trivial. At least a third of all patients in our study had some ill effect during hospitalization that was not related to the progression of any pathologic process, and $9 \%$ had a major untoward event.

Many changes have taken place in the in-hospital practice of medicine since Schimmel carried out his study at the Grace-New Haven Hospital. ${ }^{4}$ Units now routinely monitor a host of physiologic characteristics, and this practice permits earlier intervention, often with procedures that were not known two decades ago, and intervention may even occur at points in the clinical process that were previously unrecognizable. Furthermore, although continuous monitoring (as opposed to intermittent monitoring) of a characteristic may well be beneficial to the patient, such observations may also result in increased use of therapeutic procedures or drugs that inevitably carry risks as well as benefits.

The number of potent drugs in use has risen over the past two decades, while some pharmaceutical agents have been replaced by less hazardous ones. In addition, patients may be more seriously ill on admission now than they previously were, perhaps in part because of the requirements of 
Table 3 Presence or absence of complications and their severity by age, total number of drugs, and length of stay in 815 hospitalized patients*

\begin{tabular}{llll}
\hline Category & No complications & Minor complications & Major complications \\
\hline No of patients & 525 & 214 & 76 \\
Age (years) $\dagger$ & $57.8(18.2)$ & $62.7(15.7)$ & $64.0(16.1)$ \\
No of drugs used $\dagger$ & $7.3(4.7)$ & $11.7(6.4)$ & $17.1(8.4)$ \\
Length of stay (days) $\dagger$ & $7.9(8.5)$ & $13.8(11.7)$ & $19.3(16.7)$ \\
\hline *Differences were significant $(p<0.001)$ between each group for each variable except mean age of the groups with \\
minor and major complications. \\
†Mean (SD) for the patients in each group.
\end{tabular}

Table 4 Smoothed probabilities of iatrogenic complications based on data obtained at admission

\begin{tabular}{llll}
\hline \multirow{3}{*}{ Source of admission } & \multicolumn{3}{l}{ Probability of ... } \\
\cline { 3 - 4 } \cline { 3 - 3 } & House officer's assessment complication & Major complication \\
\hline \multirow{2}{*}{ Home or housing facility } & Stable to good & 0.25 & 0.04 \\
& Fair & 0.40 & 0.10 \\
Other hospital or nursing & Critical to poor & 0.46 & 0.16 \\
home & Stable to good & 0.36 & 0.10 \\
& Fair & 0.52 & 0.20 \\
& Critical to poor & 0.57 & 0.31 \\
\hline & & \\
\hline
\end{tabular}

utilization review committees. Although the data of Schimmel are not altogether comparable with ours, the risk associated with hospitalization has almost certainly not diminished in comparison with the situation 15 to 20 years ago, and the risk of a serious problem may well have increased.

Secondly, the exposure to drugs seems to be a particularly important factor in determining which patients have complications, and such exposure was also associated with increased severity of complications. It is of interest that Seidl et al noted at least one drug reaction in $17.1 \%$ of patients admitted to Johns Hopkins Hospital over a 3 month period. ${ }^{7}$ The rate in patients over 50 years old was greater than the mean attack rate (and progressively so for each decade, from $14.3 \%$ to $24.0 \%$ ). In our patients, the percentage of serious complications caused by diagnostic and therapeutic procedures was greater than that in the other two categories ("drugs" and "miscellaneous"). However, it must be noted that a number of acknowledged high-risk procedures were included in this subdivision. Information is not available on the total number of procedures (both high-risk and low-risk) that were carried out over the course of the study. Other exposure rates and their risks (for example, certain procedures or administrative practices) were not analyzed but may well be worthy of study.

Thirdly, the data reported here were obtained from a medical service at a tertiary care university hospital. The amount and kinds of iatrogenic illness occurring in other settings (for example, a surgical service or a small community hospital) may be quite different. The characteristics of the patients at admission must be important in determining these results, but other factors might also be expected to influence the findings. Experience with drugs or procedures in addition to the staffing patterns of nurses and physicians may well affect the rate of iatrogenic disease and the ability to monitor untoward occurrences.

Almost a quarter of a century after the work of Barr, ${ }^{1}$ we have demonstrated that there are still risks involved with medical care, and the risk is probably greater than ever, although such comparisons across time are difficult to make. Serious consideration should be given to new methods of monitoring untoward occurrences in hospitalized patients, especially on medical services. Attention should also be directed to educational programs about iatrogenic disease. For example, it is not unusual for the chief of the medical service at any of the major teaching hospitals to discuss at morning report the patients who were admitted in the previous 24 hours. This tradition stresses events occurring before hospitalization or during the initial diagnostic workup, but it does not seem to give sufficient emphasis to the risks associated with the diagnostic or therapeutic methods, especially drugs, that are in use during hospitalization. If the morning report included discussion of patients five to seven days after their admission, the benefits and risks of hospitalization might be more apparent.

It must be emphasized again that we are making no statement whatsoever about the relative risk and benefit of hospitalization. It may be logically sound to speculate that the benefit of hospitalization far exceeds the risk, given the severity of illness of the population in hospitals, the natural progression of their diseases, and the value of alternative modes of therapy. However, regardless of whether patients admitted to the hospital today are unusually ill, and regardless of what benefits accrue to these persons through hospitalization, mechanisms must be developed to assess the hazards of hospitalization in an ongoing manner. Technologic, educational, and administrative means can then be sought to reduce the number and severity of untoward events, and the efficacy of such efforts can be ascertained.

\section{Authors' affiliations}

K Steel, P M Gertman, C Crescenzi, J Anderson, Geriatrics Section and the Section of Health Care Research, Evans Memorial Department of Clinical Research, Department of Medicine, University Hospital, Boston University Medical Center, 720 Harrison Ave, Suite 503, Boston, MA 02118 , USA

Supported in part by a grant (5 R21 AG 01178) and a Geriatric Medicine Academic Award (AG 00060) from the National Institute on Aging, National Institutes of Health, Department of Health and Human Services. 


\section{REFERENCES}

1 Barr DP. Hazards of modern diagnosis and therapy-the price we pay. JAMA 1956;159:1452-6.

2 Moser RH. Diseases of medical progress. N Engl J Med 1956;255:606-14.

3 Kane RL. latrogenesis: just what the doctor ordered. J Community Health 1980;5: 149-58.

4 Schimmel EM. The hazards of hospitalization. Ann Intern Med 1964;60:100-10.

5 Reichel W. Complications in the care of five hundred elderly hospitalized patients. J Am Geriatr Soc 1965;13:973-81.

6 Bishop YMM, Fienberg SE, Holland PW. Discrete multivariate analysis: theory and practice. Cambridge, Mass: MIT Press, 1975.

7 Seidl LG, Thornton GF, Smith JW, et al. Studies on the epidemiology of adverse drug reactions. III. Reactions in patients on a general medical service. Bull Johns Hopkins Hosp 1966;1 19:299-315.

\section{COMMENTARY}

\section{IATROGENIC ILLNESS: A CALL FOR DECISION SUPPORT TOOLS TO REDUCE UNNECESSARY VARIATION}

Steel et al raised a red flag for the medical community in 1981 when they articulated the serious risks associated with hospitalization. ${ }^{1}$ They identified a lack of progress in the 15 years that followed a previous report of the same problem. The many advances in diagnostic and therapeutic interventions that had appeared during those 15 years were not matched by a reduction in iatrogenic illness suffered by patients in hospital. They identified the types and magnitudes of several risks without assigning blame or claiming that the iatrogenic illnesses were preventable; $36 \%$ had at least one iatrogenic illness, $9 \%$ had a major iatrogenic illness, and $2 \%$ sustained an iatrogenic illness that contributed to death.

Interestingly, iatrogenic illness occurred in several different clinical settings within the medical service they studied. One of the strengths of their study lies in the inclusion of all new patients admitted to medical and metabolic wards and to both an intensive care unit and a coronary care unit. Iatrogenic illness was encountered in all of these settings. As expected, the intensive care settings accounted for more of the iatrogenic illness than did the others. However, when subjected to a logistic analysis, the unit in which the patient received care was not a determinant of iatrogenic illness; only the referring site (home, hospital, nursing home, etc) and the assessment of the patient's condition on admission by the house officer were important determinants. This suggests that both patient and system (environment and clinician) attributes contributed to iatrogenic illness. One might be tempted to argue that the increasingly large information burden borne by clinicians is responsible for the current but not for past (at least distant past) iatrogenic illness. This seems unlikely since human cognitive ability is so strikingly limited and so small compared with the information clinicians encounter and have encountered for over a century. ${ }^{2}$ It is more likely that iatrogenic illness and other forms of errors in healthcare delivery are linked to limitations in human decision making and to defects in the healthcare delivery system. ${ }^{3}$

Steel et al sounded an appropriate and currently applicable call for a response from the medical community. They requested technological, educational, and administrative advances to meet the investigative and clinical needs of this important problem. In their discussion they consider the adverse effects of continual monitoring and point to the danger that newer monitoring techniques might lead to increased diagnostic and therapeutic interventions, both of which carry risk of iatrogenic illness. This is a prescient pointer to the extensive use of pulmonary artery catheters in the seriously ill, ${ }^{4}$ an issue under current investigation by the
National Institutes of Health/National Heart Lung and Blood Institute Acute Respiratory Distress Syndrome Network. While Steel et al are careful to avoid judgments about culpability and the risk/benefit ratio for patients admitted to hospital, they emphasize the need for further research into untoward events among hospitalized patients. They note, correctly, that patients may still benefit from health care even when they encounter important risks in so doing. We now have adequate evidence that many of these risks are unnecessary and avoidable. ${ }^{3}$

Interestingly, their method included a standardized process (study instrument) for medical record abstraction. Like many study methods, it embraced a standardized approach for obvious investigative reasons. This provides an ironic contrast with one of the causes of a subset of the iatrogenic illness they addressed-namely, those resulting from error in the clinical setting linked to the lack of clinical standardization (unnecessary variation in practice). ${ }^{5}$ Many clinicians respond openly that such standardization interferes with individualization or tailoring of treatment to the needs of a specific patient. This was the position adopted by the US Office of Human Research Protection in the recent controversy concerning the protocols used by the National Institutes of Health/National Heart Lung and Blood Institute Acute Respiratory Distress Syndrome Network. The Network argued-and was supported by the reviews of many independent experts-that individualization of treatment was maintained within the constraints of the evidence-based therapeutic protocols. ${ }^{6}$

A recent publication of the favorable impact of a checklist used in the intensive care unit to identify patient goals for all members of the clinical staff is sobering. ${ }^{7}$ This simple paper based checklist evokes images of the US Federal Aviation Agency mandated checklist used by airline and other pilots. Its implementation was followed by more successful communication and by a decrease in the length of stay in the intensive care unit. ${ }^{7}$ Other examples exist of similar strategies carried out with more complex tools. ${ }^{8}$ These more complex tools require electronic structures that could constitute barriers to implementation. ${ }^{9}{ }^{10}$ The simple paper based checklist does not. Both are examples of decision support tools effectively brought to the point of decision making.

The reduction of iatrogenic illness will depend on many factors, including the reduction of error. ${ }^{3}$ This can be achieved in part by reducing unnecessary variation in care $^{5}$ with decision support tools. ${ }^{78}$ Error reduction can also be achieved through identification and abandonment of unnecessary diagnostic and therapeutic interventions, a demanding but sorely needed activity that will require carefully conducted clinical trials. To this end, decision support tools can provide the adequately explicit methods necessary to maximize credibility of many clinical trials. ${ }^{11}{ }^{12}$

A H Morris

Pulmonary and Critical Care Divisions, Departments of Medicine, LDS Hospital and University of Utah School of Medicine, Salt Lake City, Utah 84143, USA; Idamorri@ihc.com

Supported by the NIH (RO1-HL-36787, NO1-HR-46062), the AHCPR (HS 06594) the Deseret Foundation, the Respiratory Distress Syndrome Foundation, the LDS Hospital and IHC Inc.

\section{REFERENCES}

1 Steel K, Gertman PM, Crescenzi C, et al. latrogenic illness on a general medical service at a university hospital. N EnglJ Med 1981;304:638-42.

2 Cowan $\mathrm{N}$. The magical number 4 in short-term memory: a reconsideration of mental storage capacity. Behav Brain Sci 2001;24:87-187.

3 Reason J. Human error. Cambridge, UK: Cambridge University Press, 1990.

4 Connors AFJ, Speroff T, Dawson NV, et al. The effectiveness of right heart catheterization in the initial care of critically ill patients. SUPPORT Investiaators. JAMA 1996:276:889-97. 
5 Wennb̆erg JE. Unwarranted variations in healthcare delivery: implications for academic medical centres. BMJ 2002;325:961-4

6 Steinbrook R. Trial design and patient safety-the debate continues. N Engl J Med 2003;349:629-30.

7 Pronovost PB, Dorman S, Lipsett T, et al. Improving communication in the ICU using daily goals. J Crit Care 2003;18:71-5.

8 Morris AH. Decision support and safety of clinical environments. Qual Saf Health Care 2002;11:69-75.

9 Rubenfeld G, Cooper C, Carter G, et al. Barriers to providing lung protective ventilation to patients with acute lung injury and acute respiratory distress syndrome (ALI/ARDS). Am J Respir Crit Care Med 2001;163:A767

10 Berenholtz SP, Peter J. Barriers to translating evidence into practice. Curr Opin Crit Care 2003;9:321-5.

11 Morris A. Algorithm-based decision making. In: Tobin M, ed. Principles and practice of intensive care monitoring. New York: McGraw-Hill, 1998:1355-1381.

12 The Acute Respiratory Distress Syndrome Network. Ventilation with lower tidal volumes as compared with traditional tidal volumes for acute lung injury and the acute respiratory distress syndrome. N Engl J Med 2000;342:1301-8. 